International Journal of Reproduction, Fertility \& Sexual Health (IJRFSH)

ISSN: $2377-1887$

\title{
Application of the PEN-3 Cultural Model in Assessing Factors affecting Adolescent Pregnancies in Rural Eastern Cape, South Africa
}

Nomsa R. Chemuru*, Sunitha C. Srinivas

Research Article

Department of Pharmacy Practice, Faculty of Pharmacy, Rhodes University, Grahamstown, South Africa.

\begin{abstract}
Early pregnancy and child birth increases the health risk for the mother and the baby. It is a public health concern in South Africa as it may affect the health; social and economic well-being of society at large. Although immediate determinants of teenage pregnancy in South Africa relate to behaviour, the fundamental drivers are more deeply rooted institutional problems of poverty, underdevelopment and gender based violence. In addition, government policies, socio-economic injustices, unequal power structures and culture contribute to the high rates of teenage pregnancies in South Africa. The aim of this study was to assess contributing to adolescent pregnancies with in three rural communities in Eastern Cape Province. Guided by the PEN-3 cultural model and Community-based participatory research principles, an explorative qualitative research design was undertaken with 14 community care workers from Glenmore, Ndwayana and Grahamstown. As phase one of an ongoing study, semi-structured interviews were carried out with the participants. The results were coded according to the domains of the PEN 3 model. The main findings indicated that adolescent pregnancies are a significant cause for concern in the communities under study. Perceptions (myths surrounding contraceptive use), enablers (availability of clinics), and nurturers (parents, peers and boyfriends) were found to be important in making decisions regarding contraceptive use and sexual activity amongst adolescents. These factors identified will inform the development of culturally sensitive and appropriate health promotion material during the next phase of the study.
\end{abstract}

Keywords: Adolescent Pregnancies; PEN-3 Cultural Model; Rural Communities; Health Promotion.

\section{*Corresponding Author:}

Nomsa R. Chemuru,

Department of Pharmacy Practice, Faculty of Pharmacy, Rhodes University, P. O. Box 94, Grahamstown, 6140, South Africa.

E-mail: nchemuru@yahoo.com

Received: November 05, 2015

Accepted: December 08, 2015

Published: December 11, 2015

Citation: Nomsa R. Chemuru, Sunitha C. Srinivas (2015) Application of the PEN-3 Cultural Model in Assessing Factors affecting Adolescent Pregnancies in Rural Eastern Cape, South Africa. Int J Reprod Fertil Sex Health, S1:001, 01-08. doi: http://dx.doi.org/10.19070/2377-1887SI01001

Copyright: Nomsa R. Chemuru ${ }^{\circ}$ 2015. This is an open-access article distributed under the terms of the Creative Commons Attribution License, which permits unrestricted use, distribution and reproduction in any medium, provided the original author and source are credited.

\section{Introduction}

\section{Problem statement}

The general health status of South African population appear satisfactory until the statistics are broken down by population group, socioeconomic status as well as provinces [1]. Socio-economic issues such as poverty and persistent inequalities in access to health services reflects disparities in the health status of individuals particularly in the rural areas [2]. The Eastern Cape is one of the nine provinces of South Africa and is located south-east part of the country. The Eastern Cape Province is one of the poorest provinces in South Africa in terms of average monthly household expenditure $[1,2]$. The province also has the lowest proportion of people living in formal housing $(46.9 \%)$, with access to electricity $(31.3 \%)$, and with the lowest proportion of households with tap water inside the dwelling (24.4\%). Moreover, less than $17 \%$ of the population depending on labour income and $26 \%$ of the poverty among households depending on labour income [3].

One of the health concerns associated with the low socio-economic status in the Eastern Cape is adolescent pregnancies. Adolescent pregnancy can be described as pregnancy that occurs in girls from puberty to the age of 19 [4]. Adolescent pregnancy remains public health concern in South Africa and more common amongst young African and coloured women in rural areas [2]. Over the years the highest proportion of under-18 deliveries was in the Eastern Cape and the lowest in Gauteng province. The proportion of women under the age of 18 who ever gave birth nationally was $8.2 \%, 8.0 \%, 7.0 \%$ and $7.8 \%$ in $2011,2012,2013$ and 2014 respectively. Despite the declining trend in the average birth-rate, the proportion in the Eastern Cape remains high. The proportion of women under the age of 18 who ever gave birth recorded for the Eastern Cape was 10.6\%, 10.6\%, 10.0\% and 10.1\% in 2011, 2012, 2013 and 2014 respectively [5].

\section{Consequences of unwanted adolescent pregnancies}

Early pregnancy and child birth increases the health risk for the mother and the baby and may affect the health, social and economic well-being of society at large. Adolescents who become pregnant below the age of 18 are at a higher risk of dying during childbirth than older women due to obstetric complications such 
as haemorrhage, pregnancy-related infections and complications of hypertension [6]. Complicated births such as caesarean section deliveries are also common amongst adolescents mainly due to underdeveloped pelvises which may lead to obstructed labour. In developing countries, medical services for a caesarean section may not be available resulting in infant mortality or maternal deaths [7-9]. Furthermore, adolescent mothers have an increased risk of premature and prolonged labour, miscarriages and stillbirths [10]. Infants of adolescent mothers generally have low birth weights and are at an increased risk of foetal growth retardation and perinatal deaths $[11,12]$. The socio-economic consequences of adolescent pregnancy are also massive as schooling is interrupted and early parenthood is likely to affect adversely educational achievement with significant employment and socio-economic implications $[13,14]$. Therefore efforts to reduce adolescent pregnancy are central to the promotion of women's educational, social, and economic development.

\section{Contextualizing adolescent pregnancies}

The context of adolescent pregnancies differs depending on the setting. Adolescent pregnancies may be intended or unintended. Intended pregnancies are common in adolescents in some developing countries and are usually in the context of marriage or a recognizable union [15]. In these developing countries, some girls are still expected to marry and begin childbearing in their early or middle adolescent years based on traditional practices and norms, before they are physically or mentally ready to do so [16]. In Kenya, for example, early marriage and early childbearing, especially in the rural areas, are common as adolescent girls are sanctioned and encouraged to do so by families and the wider community [17]. Intended pregnancies may also occur outside a recognizable union where young women have their first baby in the middle or late teenage years [18]. Motivations for such behaviour are different, for example in South Africa, pressure from families or male partners to prove a woman's fertility has often contributed to early childbearing as a woman cannot get married until she has had at least one child $[19,20]$. The absence of disincentives to prevent early childbearing, such as no alternatives for education or employment, has also led to adolescent pregnancies in this context [21].

Unintended pregnancies amongst adolescents are usually in the context of outside of marriage or a recognizable union, and often carry a social stigma in many communities and cultures [22]. Such pregnancies occur in adolescents who become sexually active at an early age when they do not know how to avoid unwanted pregnancies. They often lack access to sexual and reproductive health services and contraceptives that they need to avoid pregnancies [23]. Even if they have access to such services, girls and young women are often powerless to insist on their use in relationships. In South Africa, factors such as gender inequalities may contribute to unwanted adolescent pregnancy. In some communities women are placed in a subordinate position according to the societal hierarchy which leaves women with little or no negotiating power in sexual acts in a relationship [20]. Gender-based violence due to their subordinate position may also contribute to unwanted pregnancies in adolescents such that adolescent girls may be unable to refuse unwanted sex or to resist coerced sex [14, 20, 24].

Young school girls may also engage in sex with older partners in exchange for gifts or money, resulting in them having little or no negotiating power with their partners to insist on condom usage [22]. The problem of pregnancies in adolescence should be viewed within the broader socio-economic-cultural environment in which adolescents operate. For instance, lack of parental guidance and counselling on issues of sexuality and sex education is reinforced by cultural taboos that inhibit such discussions [25]. These culturally engrained practices cannot be solved by traditional sex education alone but also require culturally sensitive health promotion.

\section{Culture sensitive health promotion}

Health behaviour theories used in public health and health promotion often ascribe poor health outcomes to an individual's action or inaction or unwillingness to heed preventative health messages. Recently researchers have focused on the role of culture in health promotion by applying it to existing socio-behavioural variables in order to develop health-related interventions for positive health outcomes $[26,27]$. Health promotion activities, whether targeted at individuals or communities, are difficult tasks, because culture creates unique patterns of beliefs and perceptions of the meaning of health and these may have an impact on the outcomes of health promotion activities. Culture can be described as a system of related values, concepts, meanings and beliefs active enough to influence the judgment, communication and behaviour of an individual $[28,29]$. Culture therefore shapes perceptions and practices of individuals with regard to health and mediates responses to health information. To change health behaviours, promotion of positive health behaviours within the cultural logic of its contexts is of importance. De Walt et al. suggested that careful implementation of culturally sound methods in delivering health services has an impact in reducing the gap between culture and health promotion $[30,31]$.

\section{The PEN-3 cultural model}

The PEN-3 model examines cultural meanings such as beliefs and values of health behaviour that have gained a central location in framing people's relationships with health in social and cultural contexts. The three domains of the PEN-3 model incorporate relationships and expectations, cultural empowerment, and cultural identity. Each domain incorporates three individual constructs and the name PEN-3 is derived from the acronyms of the concepts of each of these constructs [32].

The Relationship and expectations domain focuses on the behaviour of an individual, resources and influence of family and friends in making health-related decisions but from a point of view of how cultures define the roles of persons and their expectations in the family and community relationships. The construction and interpretation of behaviour is usually based on the interaction between the perception of that behaviour, resources that enable or constrain the actions, and the influence of family, friends and social structures in nurturing the behaviour. Cultural empowerment domain is based on the belief that culture represents a range of good (Positive), indifferent (Existential) and negative (Negative), therefore interventions should focus not only on the bad influence of culture but also on promoting the good. The Cultural identity domain defines the target population and suggests that interventions can take place at an individual (Person), Extended family or Neighbourhood levels [32-34]. 


\section{Aim of the study}

The aim of this study was to assess factors could contributing to adolescent pregnancy in three rural communities in the Eastern Cape. The results in this study will inform on a strategy to implement activities aimed at preventing teenage pregnancies.

\section{Ethical considerations}

Prior permission to conduct the study was obtained from the Faculty of Pharmacy Ethics Committee, Rhodes University and the Eastern Cape Department of Health.

\section{Method}

Guided by the PEN-3 cultural model and Community based participatory principles, an explorative qualitative research design was undertaken with 13 Community Care Workers (CCWs) working with two non-governmental organisations (NGOs) in Glenmore and Ndwayana, and a field worker from Grahamstown. As the first phase of an ongoing study, semi-structured interviews were carried out with each of the participants to determine factors affecting adolescent pregnancies from a cultural point of view. The interviews were conducted by the researcher in English and a translator was used in order to accommodate participants who were not familiar with the English language or might feel more comfortable expressing themselves in isiXhosa. The translator was used to translate from English to isiXhosa, which is one of the official languages of South Africa, and vice versa. Each interview took 20-30 minutes and 14 interviews were conducted. Note-taking was used to collect data and interview responses were recorded. The voice recordings from the semi-structured interviews were translated and transcribed. The data was then managed using NVIVO ${ }^{\circledR} 2010$ and analysed according to the domains of the PEN-3 model.

\section{Results}

\section{Perceptions}

The perceptions construct of the relationship and expectations domain refers to the knowledge, attitudes, values, and beliefs affecting personal, family, and community motivation to change behaviour. Positive perceptions are the knowledge, attitudes and/or beliefs that positively influence healthy decisions with regard to early sexual debut and contraceptive use amongst adolescents and in the community. For example, knowledge of the consequences of early pregnancy may influence the age of sexual debut of an individual. Responses that negatively affected decisions about contraceptive use or knowledge and beliefs that influenced early sexual debut were categorised as negative perceptions.

Fears about contraceptive use (negative): The community care workers expressed concern about adolescents' non-adherence to contraceptive therapy, especially with regard to hormonal injections. The adolescents perceive the hormonal injection to cause a weight gain, leading to non-adherence to the contraceptive therapy.

"The problem is that they say they don't want to use contraception because they don't want their bodies to become jelly like, that's the problem. They always say no I'm using a condom, but still they're falling pregnant.'P4

The community care workers identified the male condom and the hormonal injection as the main methods of contraception used amongst the adolescents. The CCWs also reported that the female condom was unpleasant to use because of its size.

"...so the girls are scared of the female condoms, they are big so they are scared."P14

Knowledge on factors contributing to and the consequences of adolescent pregnancies (positive): From the responses of the CCWs, it was evident that CCWs had knowledge of factors contributing to adolescent pregnancies in their community, for example peer pressure.

"The main causes of teenage pregnancy is peer pressure and others do not want to come to the clinic for family planning because not that they are scared but because of peer pressure, if one stops coming to the clinic the other will not come."P10

CCWs also had knowledge of the consequences of early childbirth, for example the disruption of schooling, and the possible health effects, such as the risk of getting cervical cancer.

"I tell them that having a baby while they're still kids themselves is not great because once they have a responsibility of a child they won't be able to go to school.’P8

"When a child falls pregnant at an early age, they are at a risk of getting cervical cancer.’P9

Community concern for the welfare of teenage girls (positive/negative): The CCWs are concerned about the socio-economic welfare of teenage girls if they were to fall pregnant in their adolescent years as they perceive they are still young to be involved in sexual activity and are expected to be in school (positive). However, they also reported contrasting feelings with regard to teenage pregnancy in the community: some members of the community are concerned for the welfare of pregnant adolescents whereas some are not concerned (negative).

"I feel bad because falling pregnant at a young age makes life difficult, because now the child stops going to school in order to take care of the baby and they do not even have jobs."P6

"They still get shocked because the girl would still be young and expected to be in school.’P13

“They actually don't care, they don't really pay attention to them."P4

Knowledge on the importance of contraceptive use (positive): CCWs have knowledge of the advantages of using contraceptives amongst women and men in the community in preventing pregnancies and HIV/AIDS. They also reported that the use of contraceptives in the community was an acceptable practice which has been put into practice in their health promotion activities conducted in the community.

"Yes I talk to them, I explain to them that it is important to use a condom whenever one is engaging in sexual activities to protect against HIV/AIDS and pregnancy.’P8 
"I always encourage them to go for family planning because it's their future we are concerned about and that the first important thing in life is to go to school but they do not go for family planning and they also don't want to use condoms."P7

Early sexual debut as unacceptable(positive): From the responses of CCWs, teenagers are perceived as too young for sexual activities (positive). However, this perception does not prevent teenagers from engaging in sexual activities but prevents them from accessing contraceptive methods from the clinic.

"I think they see it [sexual activity amongst teenagers] as a problem because they don't want even a 10 year old to come here to collect condoms or 12 years old to use injection because it's early to have sex."P14

Cultural barriers related to communication about sex-related matters (negative): CCWs reported that talking about sex and sexuality in the community is generally not common as they perceive it as encouraging teenagers into early sexual debut.

"Parents are not so keen on talking about such things [sex].”P6

"Oh no ways, no one wants to talk about it [sex] in the community, even if you happen to pay someone a visit and the topic [sex] comes up and you encourage them to talk to their children about it, they say there is no way they could talk to their children about such things, if you do you would be teaching them 'wrong/bad' things."P9

\section{Enablers}

This construct refers to the cultural, societal, systematic or structural forces affecting change in an individual or the community. This applies to resource availability, access, referrals, government officials, skills and services. Positive enablers refer to resources and institutional support that are beneficial and support adolescents in making healthy decisions to prevent adolescent pregnancy, for example, the availability of and access to health services at clinics. Negative enablers are unsupportive structures for the adolescents that may facilitate risky sexual behaviour amongst the adolescents, such as media.

Availability of community care workers (positive): CCWs are community members who serve and respond to the health needs of the community. The main aim is to increase and improve the community's access to relevant health information. The CCWs conduct health promotion activities in TB, HIV/AIDS and sometimes adolescent pregnancy. When asked to describe their jobs, two of the participants responded:

"I go into the township, do home visits, when I arrive I introduce myself and tell them I'm bringing the health service to them as the government has seen that people in the community are struggling and some are being abused by their husbands or children are also being abused and they have hired us to go to the people."P12

"I look after sick people, check if children who are supposed to be in school or crèche that they actually go, to help those who eligible for social grant to get it, to get treatment from the clinic for those who are very sick and unable to get it themselves". P13
Availability of health care services (positive/negative): There is one clinic in Glenmore, one in Ndwayana, and 6 clinics in the Grahamstown area (positive). Ndwayana and Glenmore rural communities are economically less developed compared to other towns in South Africa, and access to the clinics is difficult for most community members as they live far away from the clinic and rely on donkey carts to get them to the clinics. Some of the people have to walk long distances to get health care services (negative). There are no pharmacies nearby offering health services to the Ndwayana and Glenmore communities (negative). This will impact on the motivation to visit the clinics for family planning services and may be the reason for defaulting contraceptive therapy.

The clinics are structured so that certain chronic conditions such as tuberculosis (TB), HIV/AIDS, hypertension and diabetes are only attended to on a certain day of the week. For example, Wednesday is an Antenatal Care day, and Tuesdays is an HIV patients review and patients come for their monthly medicine supplies. This structure is not convenient for adolescents who want to come and get condoms or other contraceptives as there is no day set for family planning. Adolescents are scared of the stigma associated with the different conditions, especially HIV/AIDS (negative).

"Teenagers are not free to go to the clinic for anything whether it is contraceptives or in general if they are sick because of the days that are scheduled for different chronic illnesses eg hypertension, diabetes and HIV. Tuesday is set aside for HIV and Wednesday for ANC therefore going on those days means you are pregnant or have HIV, and its not fair, the support structures in the clinics is not helping."P1

Access to contraceptives (positive): Different contraceptive methods are offered at the clinics, namely condoms, hormonal injection, birth control tablets and sub-dermal hormonal implant. The community care workers identified the male condom and the hormonal injection as the common methods of contraception amongst the adolescents. These methods are freely available at the clinics, and sometimes condoms are distributed by CCWs when they visit the communities. However the CCWs reported that only a few adolescents request condoms.

"Every child comes and takes the condoms from there [pointing at the reception area], in front it's not a problem...There are only a few that come, you see the same faces all the time most of them do not actually come."P2

Health promotion activities in the community (positive/ negative): Health promotion activities organised by loveLife and the Ubunye Foundation are conducted in the community hall with the help of CCWs. These activities are aimed at educating the communities on health-related issues such as TB and HIV (positive).

"At school they do talk about sex, there's also someone from loveLife, he also comes and talks to them about such things.'P6

However, there is a lack of health promotion materials developed for the prevention of adolescent pregnancy, and if available there are in English which most people do not comprehend as they are isiXhosa first language speakers (negative). 
"In my opinion or suggestion, I was thinking this booklet is meant for teenagers but even in those teenagers others are not good in English, so I was thinking of what about you write in English and isiXhosa even two piece booklets... even us as ccw some it is difficult to read in English if I read in isiXhosa it will be easier to explain to teenagers but if you give me this English book even me I do not understand what is here and they will be asking me questions so it will be difficult for me to answer because I do not understand."'P14

Child Support Grant (negative): The CCWs identified the Child Support Grant (CSG) as a contributory factor to adolescent pregnancy. The government implementation of the CSG has a negative influence on teenagers as they willingly fall pregnant to receive the monetary stipend every month.

"They actually want the social grant, so they do fall pregnant deliberately.’P10

"Another thing is the social grant, they think that if one has a baby and therefore get the social grant, they actually have money."P8

Religion (negative/positive): Most of the community members in Grahamstown, Glenmore and Ndwayana are members of mostly Christian denominations. The church as an institution is an enabler and church doctrines and norms fall under the nurturer construct because of the influence on the individual, family and the community. It is further classified as both positive and negative under the community empowerment domain. Under certain doctrines, sex before marriage is forbidden which influences how children are expected to behave (positive). However some doctrines do not allow the use of contraceptives or sex education in any way, which may hinder the use of contraceptives amongst the adolescents, and even if they wanted to they may not have enough information (negative).

"We would like to talk about prevention of pregnancy but it is difficult because of religious beliefs that you are not supposed to talk about sex until you get married or preventing pregnancy.”P1

Socio-economic status (negative): As described by the CCWs, the three communities are characterised by poor socioeconomic conditions such as unemployment and poverty. The CCWs suggested that most adolescent pregnancies were from children being taken care of by a single parent or their parents were adolescents when they gave birth to their children. The socio-economic status of the community was also identified as a contributory factor to adolescent pregnancy as adolescents sought financial security and support from older men resulting in cross-generational relationships. This also contributed directly to adolescents willingly falling pregnant in order to receive the child support grant.

"Most of these kids usually date people who are older than them, so I would say for some of them it is poverty related. They date these older men because they need money."P7

"Well it concerns me because you find that the child is quite young and is from a very disadvantaged family where no one works and they struggle to even buy food.’P13

Media (negative): The CCWs identified the negative impacts of media on contributing to adolescent pregnancy. They suggested that what children see on the television, magazines and the internet influences the behaviour and practices of adolescents.
"Some of them see it [sex] on TV and then they want to try it out."P6

Access to shebeens(negative): The communities have no organised instructive activity during their free time, such as during weekends and holidays, which make them vulnerable to activities that expose them to adolescent pregnancy. One of the activities undertaken by adolescents is going to shebeens where they are exposed to older men and alcohol.

"Well on weekends, even this football thing is new because they don't usually have anything [to do]. But they do go to these nice things, to Shebeens or Tavern, even the underage ones go.”P3

\section{Nurturers}

This construct refers to the degree to which attitudes, beliefs and actions are influenced, mediated and nurtured by extended family, kin, friends and the community. Responses which showed support from family members and friends regarding healthy sexual decisions were classified as positive nurturers. Negative nurturers are the unsupportive partners, family members and significant others who negatively influence healthy sexual behaviour. The main people that were found to have significant influence on the community are parents, friends and community care workers.

Parents (negative): The CCWs identified that a lack of parental supervision on children's activities and their actions significantly influence the behaviours of children. One of the respondents said:

"I think that another cause lies with the parents, because they are also young parents and are not as responsible. For instance I have my child, sometimes let's say I will go out at night and sleep at my boyfriend's/husband place. During that time, there is no one to watch her and to ensure that she sleeps at home. I can take care of her during the day and ensure that she is at home but at night if I go out, then no one does. I think that's where the problem lies, because these children are also parented by young parents as well.’P5

"It makes me wonder if anyone cares at home. Because as a parent you have to be there, you have to show that you care.’P12

The role of parents in the community as perceived by the CCWs is to inform and educate adolescents on issues regarding sexuality and reproductive health. However, the CCWs expressed concern about parents not educating their children as 'sex talk' is not an easy topic to discuss or they are too shy. Despite their concern, some mothers still talk to their children about sex in spite of cultural restrictions.

"Parents are not so keen on talking about such things [sex]."P6

"Even if you happen to pay someone a visit and the topic comes up and you encourage them to talk to their children about it, they say there is no way they could talk to their children about such things [sex], if you do you would be teaching them 'wrong/bad' things."P9

Due to the socio-economic status of the communities, the social setup in terms of the living conditions can affect the behaviour of the child: 
"Most children do not live with their fathers and mothers and may have boyfriends who come home with and the children see these things and also want to practice it; the houses are small therefore they share rooms with parents and also want to practice what their parents are doing and most parents do not think of that.'P1

Friends/peers (negative): The CCWs identified friends (peer pressure) as having a great influence on the decisions made by adolescents with regard to contraceptive use as well as in sexual debut. They noticed a trend in peer groups where an individual had fallen pregnant and her friends would also fall pregnant. Within these peer groups, the CCWs identified difference in age groups resulting in the young girls imitating the behaviours of the older groups.

"I think it's peer pressure or some form of competition. Because once one of them falls pregnant, the others follow suit."P10

"Children become friends with people from a different age group, older girls and they all party together.'P5

Partners/boyfriends (negative): Most children engage in sexual activity with older men or boys in order to get financial security. This may lead to coerced sex or obligatory sex to return the favour.

"Most of these kids usually date people who are older than them, so I would say for some of them it is poverty related. They date these older men because they need money.'P7

\section{Discussion}

Adolescent pregnancy is still a public health concern in South Africa, and the study explored factors affecting adolescent pregnancy in rural communities from a cultural point of view. The main findings indicated that adolescent pregnancy is a significant cause for concern in the communities under study and identified health seeking behaviours with respect to contraceptive use, availability of sexual and reproductive health services and influence of family and peers as the dominant factors contributing to adolescent pregnancies. Perceptions such as fears of weight gain due to hormonal contraceptive use and the size of the female condoms had a negative influence on the use of contraceptives amongst adolescents. These perceptions are comparable to those that emerged from studies on factors influencing non-utilisation of contraceptives in rural communities in South Africa $[35,36]$. Ramathuba et al. [36] identified that teenage pregnancies were a result of a lack of knowledge about contraception and misconceptions such as weight gain, infertility and watery discharge. The condom was cited by the majority as their preferred contraceptive method, whereas the hormonal contraceptives (the injection and the pill) were least preferred. The overall result is low contraceptive use amongst adolescents leading to increased rates of teenage pregnancies.

Conventional theories of behaviour change often focus on individual perceptions to promote change. The PEN-3 cultural model, however, focuses on the impact of culture on health beliefs and actions, and proposes that public health and health promotion should focus not only on the individual, but also on the cultural context that nurtures a person's health behaviour in his or her family and community [32, 33]. The model was therefore used to identify factors contributing to adolescent pregnancies in the socio-economic-cultural context in which they occur. From the results, decisions about contraceptive use are affected by the lack of access to health services and the influence of family, friends and other stakeholders in the community. The current study also showed that although clinics provide free contraceptives to the community and community care workers encourage preventing early childbearing, the decision to use contraceptives and debut of sexual activity is influenced by the distances from the clinics to their homes and the health services provided. This concurs with the results reported by Ritcher and Mlambo [37] that in rural communities, although clinics are available, transport to the health services becomes a barrier to the accessibility of the health services. In addition to lack of access to health services, there was a lack of health promotion material such as pamphlets in the appropriate language, leading to lack of access to information regarding sexual and reproductive health. The current study identified that the health promotion material was available in English which the adolescents and the local community members could not understand. Ritcher and Mlambo [37] also identified language on written health education material as a barrier to information. Halcomb et al. [38] suggested that care should be taken to make health education material appropriate to fulfil health information needs of a community such as the use of a local language and illustrations to depict certain messages.

Cultural barriers related to communication about sex-related matters have been reported as a major obstacle in having open discussions on sexual and reproductive health in rural communities by Lebese [39]. The study showed that cultural values and norms hindered sexual health dialogue in communities. It also revealed that parents use indirect language to communicate sexual health with their children. These barriers result in adolescents lacking the information they need to prevent unwanted pregnancies. These findings concur with the results found in the current study, as the CCWs identified that most parents perceive that talking to children about sex-related issues promotes promiscuity amongst adolescents and were not willing to talk to their children about sex-related issues.

The child support grant was introduced by the government to support economically vulnerable and disadvantaged children. This grant is payable to a person who takes primary responsibility for the daily needs of a child and need not necessarily be related to the child [40]. The CCWs reported that adolescents fall pregnant deliberately in order to access the CSG for financial support due to their socioeconomic background. Similarly, Kanku and Mash [41] reported that child support grants were seen as a means of increasing household incomes resulting in teenage pregnancies. However, Makiwane and Udjo [42] concluded that there was no association between the introduction of the Child Support Grant and an increase in adolescent pregnancy in South Africa. The issue of the influence of the child support grant is still debatable and requires further research.

The current study identified that health-seeking behaviour and teenage pregnancy are influenced by a collectivist orientation rather than individualist one as it identified the role of parents influencing child behaviour. A study in eastern Uganda related teenage pregnancy not so much to the sexual practices of teenagers as to the failure of girls' parents and guardians to educate children on sexual and reproductive health issues, which correlates to the results found in this study [43]. Similarly Scarinci et al. [44] highlighted importance of parents and family members influence 
in smoking cessation and smoking-prompting behaviour in initiation.

The PEN-3 cultural model was developed by Airhihenbuwa in 1989 to emphasize the need for cultural appropriateness in developing, implementing and evaluating public health interventions. Cultural context provides a clearer lens through which researchers may view and understand behaviour while highlighting important variables in future intervention design. Adolescent pregnancy is a socially embedded phenomenon and attitudes and actions of adolescents are shaped by the environment in which they exist. Interventions aimed at reducing adolescent pregnancy in any given community should address the broader social environment and social norms. Interventions for the reduction and prevention of adolescent pregnancy should be tailored with a sociocultural orientation as well as provision of sexual and reproductive health services.

\section{Conclusion}

The study was able to identify the socio-economic and cultural aspects contributing to adolescent pregnancies in these rural communities. These included perceptions about contraceptive use, availability of sexual and reproductive health services and influence of family and peers as the main contributing factors. The PEN-3 model provided a framework to assess needs for designing culturally sensitive interventions aimed at preventing adolescent pregnancy. The factors identified will be addressed in the development of culturally sensitive and appropriate health promotion materials through a participatory approach with community care workers.

\section{Acknowledgements}

The authors would like to acknowledge Rhodes University for Sandisa Imbewu Fund granted to Prof Srinivas and Ms Hornby, the Ubunye Foundation and St Mary's Development and Care Centre.

\section{References}

[1]. Bradshaw D, Masiteng K, Nannan N (2000) Health status and determinants. South African Health Review 2000: 89-124.

[2]. Pelser AJ (2012) The health, environment and development nexus in South Africa. In Health and Health Care in South Africa. Van Schaik, Pretoria.

[3]. State of poverty and its manifestation in the nine provinces of South Africa (2014) National Development Agency. http://www.nda.org.za/docs/ Research\%20Report\%20-\%20State\%20of\%20poverty\%20in\%209\%20 provinces $\% 20$ of $\% 20$ SA.PDF

[4]. World Health Organisation (2008) Fact sheet on teenage pregnancy. http:// www.who.int/mediacentre/factsheets/fs364/en/.

[5]. District Health Barometer (2014) Health Systems Trust. http://www.hst.org. za/publications/district-health-barometer-201314

[6]. Mayor S (2004) Pregnancy and childbirth are leading causes of death in teenage girls in developing countries. BMJ 328(7449): 1152.

[7]. Zabin LS, Kiragu K (1998) The Health Consequences of Adolescent Sexual and Fertility Behavior in Sub-Saharan Africa. Studies in Family Planning 29(2): 210-232.

[8]. Hoque M, Hoque S (2010) A comparison of obstetrics and perinatal outcomes of teenagers and older women : experiences from rural South Africa : original research. African Primary Health Care and Family Medicine 2(1): $1-5$.

[9]. Hoque M, Hoque S (2010) A comparative study of pregnancy complications and outcomes for the years 1999 and 2004 at a rural hospital in South Africa : implications for antenatal care. African Primary Health Care and Family Medicine.

[10]. Kumar A, Singh T, Basu S, Pandey S, Bhargava V (2007) Outcome of teenage pregnancy. Indian J Pediatr 74(10): 927-931.
[11]. Bhadauria S, Singh S, Sarkar B (1991) Teenage pregnancy: a retrospective study. J Obstet Gynaecol 41(4): 454-456.

[12]. Jolly MC, Sebire N, Harris J, Robinson S, Regan L (2000) Obstetric risk of pregnancy in women less than 18 years old. Obstet Gynecol 96(6): 962-966.

[13]. Panday S, Makiwane M, Ranchod C, Letsoalo T (2009) Adolescent pregnancy in South Africa - with a specific focus on school-going learners. Child, Youth, Family and Social Development, Human Sciences Research Council, Department of Basic Education, Pretoria.

[14]. Bhana D, Mcambi SJ (2013) When schoolgirls become mothers: Reflections from a selected group of teenage girls in Durban. Perspectives in Education 31(1): 11-19.

[15]. Engstrom L (1978) Teenage pregnancy in developing countries. Journal of Biosocial Science 10(S5): 117-126.

[16]. Fathalla MF, Sinding SW, Rosenfield A, Fathalla MM (2006) Sexual and reproductive health for all: a call for action. The Lancet 368(9552): 2095 2100

[17]. Were M (2007) Determinants of teenage pregnancies: The case of Busia District in Kenya. Econ Hum Biol 5(2): 322-339.

[18]. World Health Organisation.Guidelines on Preventing Early Pregnancy and Poor Reproductive Outcomes Among Adolescents in Developing Countries (2011).http://whqlibdoc.who.int/publications/2011/9789241502214_eng. pdf?ua $=1$.

[19]. Macleod C (1999) The "Causes" of Adolescent Pregnancy: Review of South African Research. South African Journal of Psychology 29(1): 8-16.

[20]. Jewkes R, Morrell R, Christofides N (2009) Empowering teenagers to prevent pregnancy: lessons from South Africa. Cult Health Sex 11(7): 675-688.

[21]. Mchunu G, Peltzer K, Tutshana B, Seutlwadi L (2012) Adolescent pregnancy and associated factors in South African youth. Afr Health Sci 12(4): 426-434.

[22]. Luke N (2005) Confronting the "Sugar Daddy" stereotype: age and economic asymmetries and risky sexual behaviour in urban Kenya. Int Fam Plan Perspect 31(1): 6-14.

[23]. Macleod C (1999) Teenage Pregnancy and its "Negative" Consequences: Review of South African Research - Part 1. South African Journal of Psychology 29(1): 1-7.

[24]. Wood K, Maforah F, Jewkes R (1998) "He forced me to love him": putting violence on adolescent sexual health agendas. Soc Sci Med 47(2): 233-242.

[25]. Petersen J, Atkinson P, Petrie S, Gibin M, Ashby D, et al. (2009) Teenage pregnancy-New tools to support local health campaigns. Health Place 15(1): 300-307.

[26]. Huff RM, Kline MV (1999) Promoting Health in Multicultural Populations: A Handbook for Practitioners. SAGE Publications, California.

[27]. Macdonald TH (1998) Rethinking Health Promotion: A Global Approach. Routledge, London.

[28]. Kline NK (2007) Cultural Sensitivity and Health Promotion: Assessing Breast Cancer Education Pamphlets Designed for African American Women. Health Commun 21(1): 85-96.

[29]. Helman CG (1990) Culture, health and illness: an introduction for health professionals. (2 $2^{\text {nd }}$ edtn), John Wright and Sons, Bristol.

[30]. DeWalt DA, Broucksou KA, Hawk V, Brach C, Hink A, et al. (2011) Developing and testing the health literacy universal precautions toolkit. Nurs Outlook 59(2): 85-94.

[31]. Epstein TS (1982) The social context of education and health. Health Policy Educ 3(1): 71-90.

[32]. Airhihenbuwa CO (1995) Health and culture: Beyond the western paradigm. Sage Publications, California.

[33]. Airhihenbuwa CO, Webster JD (2004) Culture and African contexts of HIV/AIDS prevention, care and support. SAHARA-J 1(1): 4-13.

[34]. Melancon J, Oomen J, Del Rincon LM (2009) Using the PEN-3 Model to Assess Knowledge, Attitudes, and Beliefs about Diabetes Type 2 among Mexican American and Mexican Native Men and Women in North Texas. International Electronic Journal of Health Education 12: 203-221.

[35]. Onyensoh O, Govender I, Tumbo J (2013) Knowledge of, attitudes towards, and practices of contraception in high school pupils in Tswaing subdistrict, North West province. Southern African Journal of Epidemiology and Infection 28(4): 227-232.

[36]. Ramathuba DU, Khoza LB, Netshikweta ML (2012) Knowledge, attitudes and practice of secondary school girls towards contraception in Limpopo Province. Curationis 35(1): E1-7.

[37]. Ritcher MS, Mlambo GT (2005) Perceptions of rural teenagers on teenage pregnancy. Health SA Gesondheid 10(2): 61-70.

[38]. Halcomb EJ, Gholizadeh L, Digiacomo M, Phillips J, Davidson PM (2007) Literature review: Considerations in undertaking focus group research with culturally and linguistically diverse groups. J Clin Nurs 16(6): 1000-1011.

[39]. Lebese R (2010) The role of culture in sexual health dialogue: an issue in the fight against sexually transmitted infections including HIV and AIDS. African Journal of Indigenous Knowledge Systems 9(2): 238-252.

[40]. Manamela T (2004) Children of poverty and child benefits in South Africa. 
SA Public Law 19(1): 161-179.

[41]. Kanku T, Mash R (2010) Attitudes, perceptions and understanding amongst teenagers regarding adolescent pregnancy, sexuality and contraception in Taung. South African Family Practice 52(6): 563-572.

[42]. Makiwane M, Udjo E (2006) Is the Child Support Grant Associated with an Increase in Adolescent Fertility in South Africa? Evidence from National Surveys and Administrative Data. Human Sciences Research Council, Pretoria.

[43]. Sekiwunga R, Whyte SR (2009) Poor parenting : teenagers' views on adolescent pregnancies in eastern Uganda : original research article. African Journal of Reproductive Health 13(4): 113-128.

[44]. Scarinci IC, Bandura L, Hidalgo B, Cherrington A (2012) Development of a Theory-Based (PEN-3 and Health Belief Model), Culturally Relevant Intervention on Cervical Cancer Prevention Among Latina Immigrants Using Intervention Mapping. Health Promot Pract 13(1): 29-40.

\section{Special Issue on}

"Pregnancy and its complications"

Edited by:

Chao Tong, Chongqing Medical University, China. E-mail: chaotongcqmu@163.com 